\title{
The benefits of advanced risk reclassification
}

\author{
Parham Eshtehardi, MD, ${ }^{\mathrm{a}}$ Ijeoma Isiadinso, $M D,{ }^{\mathrm{a}}$ and Leslee J. Shaw, $\mathrm{PhD}^{\mathrm{a}}$ \\ a Division of Cardiology, Emory Clinical Cardiovascular Research Institute (ECCRI), Emory \\ University School of Medicine, Atlanta, GA
}

Received Aug 20, 2015; accepted Aug 20, 2015

doi: $10.1007 / \mathrm{s} 12350-015-0282-5$

\section{See related article, pp. 366-378}

In years past, the value of detecting risk was measured largely by examining a clear separation among survival curves for key patient subgroups. ${ }^{1}$ In 2008 , prognostic modeling was advanced with the introduction of risk reclassification analyses which allow one to calculate the incremental improvement in risk quantification for low- and high-risk individuals based on the addition of new markers to a risk-prediction model. ${ }^{2} \mathrm{~A}$ key clinical benefit to the net reclassification improvement (NRI) analyses is that the results estimate the proportion of low- and high-risk individuals that are reclassified when two prognostic models are compared. This approach has now been successfully applied in a number of prognostic analyses using nuclear imaging. ${ }^{3-9}$

Although most reports quantifying the overall NRI have reported that nuclear variables add considerably to the ability to accurately detect low- and high-risk individuals, in this issue of the journal, Koh and colleagues ${ }^{7}$ reported intriguing results from a relatively large, singlecenter database of 6702 patients referred for exercise myocardial perfusion imaging (MPI) for either suspected or known coronary artery disease (CAD). This report provided a unique approach by formulating three models including the base comparator of a clinical model which was then compared to a model adding the Duke Treadmill Score (DTS), and then a third model adding the summed difference score and a categorical post-stress left ventricular ejection fraction variables.

Reprint requests: Leslee J. Shaw, PhD, Division of Cardiology, Emory Clinical Cardiovascular Research Institute (ECCRI), Emory University School of Medicine, 1462 Clifton Rd NE, Room 529, Atlanta, GA 30324; lshaw3@emory.edu

J Nucl Cardiol 2016;23:384-6.

$1071-3581 / \$ 34.00$

Copyright (C) 2015 American Society of Nuclear Cardiology.
This approach is interesting as it provides some insight into the comparative utility of exercise testing information alone compared with nuclear imaging. This analysis revealed that in patients with suspected coronary artery disease, the addition of DTS provided significant improvement in the NRI beyond clinical data alone; with an NRI of 0.12 (or $12 \%, P=0.019$ ). However, in this lower-risk patient subset, MPI variables did not result in a significant improvement in the NRI calculation when added to DTS and clinical variables $(P=0.9)$. These results are in line with prior reports in patients with low cardiovascular risk. A prior multicenter series reported no improvement in risk estimation in women $<55$ years of age using pharmacologic stress myocardial perfusion Rb-82 PET. ${ }^{6}$ In the paper by Kohl et al, the primary endpoint for analysis was all-cause death. One may argue that more cardiacspecific outcomes (such as nonfatal MI, cardiac arrhythmias, need for percutaneous or surgical revascularization, and hospitalization for heart failure) may yield different results. These findings are similar to the results from the WOMEN (What is the Optimal Method for Ischemia Evaluation in Women) trial which demonstrated that ETT was an effective initial diagnostic test in the evaluation of low-risk, functional women with suspected CAD. In the WOMEN trial, 824 symptomatic women with suspected CAD were randomized to either ETT or exercise MPI. There was no difference in the 2-year MACE-free survival event rates between the two groups $(P=0.59) .{ }^{10}$ The lack of significant improvement in the NRI in lower-risk cohorts is also supported by current guidelines which recommended exercise testing (without imaging) as the index strategy for evaluation of suspected ischemic symptoms in patients with low to intermediate pretest likelihood of CAD. ${ }^{11}$

However, in patients with prior CAD, the $\mathrm{Koh}^{7}$ report noted that the NRI was 0.26 (or $26 \%, P<0.05$ ) when MPI variables were added to clinical variables and DTS, a value similar to other published reports. ${ }^{3}$ Importantly, the clinical model only included a few 
variables (age, male sex, and a variable indicating $>2$ risk factors) which would magnify the added contribution of nuclear data for a second comparative model. From the Multicenter PET Prognosis registry, Dorbala ${ }^{5}$ formulated a clinical model that included 12 variables (including risk factors, symptoms, beta blocker use, and heart rate; to name a few). In the PET report, the NRI for inclusion of the percentage of ischemic and scarred myocardium on stress PET imaging for estimating cardiac death was 0.12 (or 12\%). Higher NRI calculations for stress PET imaging data estimating cardiac mortality have been reported for men (NRI: 0.17) and older women (NRI: 0.21). ${ }^{6}$ Thus, one must take care to examine what is being compared between the two models. When weak or only a few clinical variables are added to a model, this may result in a substantially higher NRI than what would be observed when a rigorous, exhaustive list of clinical variables is applied in a comparative prognostic model.

The $\mathrm{Koh}^{7}$ report provided a unique approach by formulating three models including the base comparator of a clinical model compared to a model adding the DTS, and then a third model of MPI variables. This demonstrates the incremental value of adding the DTS or data derived from MPI to baseline clinical risk factors in risk assessment. This study provides insight into which patient population can undergo exercise treadmill testing (ETT) as an initial diagnostic strategy in the evaluation of symptoms suggestive of CAD. A similar approach was employed in a recent report of 988 lowrisk patients undergoing coronary calcium and exercise myocardial perfusion SPECT. ${ }^{8}$ This report noted that when exercise testing and myocardial perfusion SPECT data were added to a model including the Framingham risk score, there was a significant improvement in the NRI; with values of $9.6 \%$ for the Duke treadmill score and $14.7 \%$ for nuclear imaging data. ${ }^{8}$ One key difference between these two reports is that the latter series had more substantial follow-up (median of 6.9 years) compared to 2.5 years in the $\mathrm{Koh}^{7}$ series. Longer-term follow-up findings can often yield varied findings as to the effectiveness of prognostication, in particular in lower-risk patient cohorts. ${ }^{12}$

In addition to the length of follow-up, there remain several aspects of an NRI analysis that are ill defined. The NRI analysis requires that discrete categories of outcome rates are clearly defined. For example, in the $\mathrm{Koh}^{7}$ series, the all-cause death rates were categorized as $<1 \%, 1-3 \%$, and $>3 \%$ per year. Similar cutpoints were used in a PET analysis estimating cardiac mortality. ${ }^{6}$ In another study, cardiac event category rates of $<6 \%$, $6-20 \%$, and $>20 \%$ were applied in an NRI analysis. ${ }^{8}$ Thus, one may envision that if outcome rates are set low, then it can be difficult for these patients to be reclassified into higher-risk categories even after the addition of other variables. By comparison, by setting the higher category at too low a risk threshold (e.g., $>3 \%$ vs $>6 \%$ ), then improvement in risk may be fraught with challenges. This latter point is particularly poignant when the duration of follow-up is short.

Future reports should perform a range of cutpoints for an NRI analysis, as none are currently defined as optimal. Likely, a pattern exists where the NRI result is dependent on the length of follow-up, the pretest risk in the patient subset, the primary outcome of interest, as well as the predefined categories of risk. If one can vary each of the components of an NRI analysis, then it would be helpful to set standards of acceptability to ensure interpretation of the results remains clinically meaningful. What is clear from the $\mathrm{Koh}^{7}$ series is that testing of lower-risk patients using MPI does not improve on near-term risk reclassification.

Beyond MPI, reports have noted an added NRI for coronary flow reserve (NRI: $17.1 \%$ for diabetics and $21.4 \%$ for non-diabetics) ${ }^{9}$ and for the heart rate change with pharmacologic stress (NRI: $18.5 \%) .{ }^{13}$ By comparison, the extent and severity of ischemia and post-stress left ventricular function added considerably to risk reclassification of patients with known CAD. These results are consistent with the abundant research supporting current approaches of ischemia-guided management as effective and at the heart of clinical practice guidelines. ${ }^{11}$

As we boldly strive to address new research questions, it remains vital to frame the work and tailor the findings for optimal cohorts. The $\mathrm{Koh}^{7}$ series reminds us that the use of stress MPI should be used in appropriately selected groups. At the heart of any diagnostic test is to select the best test that will provide additional information impacting management and improving clinical outcomes while minimizing cost and patient risk. The first step in this process is determining an individual's pretest likelihood of disease. As seen in this study, and supported by widely accepted guidelines, low-to-intermediate risk patients with suspected CAD, adequate functional capacity, and an interpretable resting electrocardiogram can appropriately be referred for an ETT to provide further risk stratification and evaluation of symptoms. MPI provides significant prognostic information when added to clinical history especially in high-risk patients. Many times, clinical researchers hope for positive findings and remain afraid of negative results. We in the field of cardiovascular imaging should embrace knowledge-negative or positive-but that which is in the best interest of our patients. This is by definition patient-centered imaging where our intentions and goals for imaging are oriented toward what is best for the patient. We encourage researchers in the field of nuclear cardiology to further define our clinical benefit 
and our limitations to testing. It is only by knowing our limits that we understand our strengths!

\section{Disclosure}

No financial conflicts to disclose.

\section{References}

1. Shaw LJ, Iskandrian AE. Prognostic value of gated myocardial perfusion SPECT. J Nucl Cardiol 2004;11:171-85.

2. Pencina MJ, D'Agostino RB, Sr, D'Agostino RB, Jr, Vasan RS. Evaluating the added predictive ability of a new marker: From area under the ROC curve to reclassification and beyond. Stat Med 2008;27:157-72 discussion 207-12.

3. Shaw LJ, Wilson PW, Hachamovitch R, Hendel RC, Borges-Neto $\mathrm{S}$, Berman DS. Improved near-term coronary artery disease risk classification with gated stress myocardial perfusion SPECT. JACC Cardiovasc Imaging 2010;3:1139-48.

4. Shaw LJ. The new era of risk reclassification in cardiovascular imaging. J Nucl Cardiol 2011;18:536-7.

5. Dorbala S, Di Carli MF, Beanlands RS, Merhige ME, Williams BA, Veledar E, et al. Prognostic value of stress myocardial perfusion positron emission tomography: Results from a multicenter observational registry. J Am Coll Cardiol 2013;61:176-84.

6. Kay J, Dorbala S, Goyal A, Fazel R, Di Carli MF, Einstein AJ, et al. Influence of sex on risk stratification with stress myocardial perfusion $\mathrm{Rb}-82$ positron emission tomography: Results from the PET (Positron Emission Tomography) Prognosis Multicenter Registry. J Am Coll Cardiol 2013;62:1866-76.

7. Koh AS, Gao F, Chin CT, Keng FYJ, Tan R-S, Chu TSJ. Differential risk reclassification improvement by exercise testing and myocardial perfusion imaging in patients with suspected and known coronary artery disease. J Nucl Cardiol 2015.

8. Chang SM, Nabi F, Xu J, Pratt CM, Mahmarian AC, Frias ME, et al. Value of CACS compared with ETT and myocardial perfusion imaging for predicting long-term cardiac outcome in asymptomatic and symptomatic patients at low risk for coronary disease: Clinical implications in a multimodality imaging world. JACC Cardiovasc Imaging 2015;8:134-44.

9. Murthy VL, Naya M, Foster CR, Gaber M, Hainer J, Klein J, et al. Association between coronary vascular dysfunction and cardiac mortality in patients with and without diabetes mellitus. Circulation 2012;126:1858-68.

10. Shaw LJ, Mieres JH, Hendel RH, Boden WE, Gulati M, Veledar E, et al. Comparative effectiveness of exercise electrocardiography with or without myocardial perfusion single photon emission computed tomography in women with suspected coronary artery disease: Results from the What Is the Optimal Method for Ischemia Evaluation in Women (WOMEN) trial. Circulation 2011;124:1239-49.

11. Fihn SD, Gardin JM, Abrams J, Berra K, Blankenship JC, Dallas $\mathrm{AP}$, et al. 2012 ACCF/AHA/ACP/AATS/PCNA/SCAI/STS Guideline for the diagnosis and management of patients with stable ischemic heart disease: A report of the American College of Cardiology Foundation/American Heart Association Task Force on Practice Guidelines, and the American College of Physicians, American Association for Thoracic Surgery, Preventive Cardiovascular Nurses Association, Society for Cardiovascular Angiography and Interventions, and Society of Thoracic Surgeons. J Am Coll Cardiol 2012;60:e44-164.

12. Shaw LJ, Giambrone AE, Blaha MJ, Knapper JT, Berman DS, Bellam N, et al. Long-term prognosis after coronary artery calcification testing in asymptomatic patients: A cohort study. Ann Intern Med 2015;163:14-21.

13. Bellam N, Veledar E, Dorbala S, Di Carli MF, Shah S, Eapen D, et al. Prognostic significance of impaired chronotropic response to pharmacologic stress Rb-82 PET. J Nucl Cardiol 2014;21:233-44. 